\title{
Brain and cognitive correlates of subjective cognitive decline-plus features in a population-based cohort
}

\author{
Gonzalo Sánchez-Benavides ${ }^{1,2}$, Oriol Grau-Rivera', Marc Suárez-Calvet ${ }^{1}$, Carolina Minguillon $^{1,2}$, Raffaele Cacciaglia', \\ Nina Gramunt ${ }^{1,2}$, Carles Falcon ${ }^{1,3}$, ALFA Study, Juan Domingo Gispert ${ }^{1,3,4}$ and José Luis Molinuevo $0^{1,2^{*}}$
}

\begin{abstract}
Background: Subjective cognitive decline (SCD) consists of self-perceived decline in cognition over time. The occurrence of specific additional features in SCD (so-called SCDplus) confers a higher risk of future cognitive decline. However, it is not known whether SCDplus patients have a distinct cognitive and neuroimaging profile. Therefore, we aimed to study the associations between SCDplus features and cognitive and neuroimaging profiles in a population-based cohort.

Methods: A total of 2670 individuals from the ALFA cohort underwent clinical, cognitive, and MRI $(n=532)$ explorations. Subjects were classified as self-reporting cognitive decline (SCD) or not self-reporting cognitive decline (non-SCD). Within the SCD group, participants were also classified according to the number of SCDplus features they met (SCD,$+>3 ;$ SCD-, $\leq 3$ ).

Results: The prevalence of SCD in the cohort was 21.4\% (55.8\% SCD-, 44.2\% SCD+). SCD+ subjects performed worse than non-SCD and SCD- subjects in memory and executive function. Among the SCDplus features, confirmation of decline by an informant was the best predictor of worse cognitive performance and lower gray matter volumes.

Conclusions: Our findings show that individuals with SCDplus features have a distinct cognitive and brain volumetric profile similar to that found in Alzheimer's disease and therefore support the use of the SCDplus concept as an enrichment criterion in population-based cohorts.
\end{abstract}

Keywords: Subjective cognitive decline, Memory, Voxel-based morphometry

\section{Introduction}

Subjective cognitive decline (SCD) has been suggested to be an initial manifestation of brain changes related to Alzheimer's disease (AD) pathology [1]. SCD has been defined as a self-experienced persistent decline in cognitive capacity in comparison with a previously normal status and not related to an acute event $[1,2]$ and is supposed to precede mild cognitive impairment (MCI), characterized by objective cognitive impairment. Objective cognitive performance in SCD is by definition within normal ranges. The use of standardized tests has been shown to have limited ability to capture differences in performance between groups with

\footnotetext{
* Correspondence: jlmolinuevo@barcelonabeta.org

'Barcelonaßeta Brain Research Center, Pasqual Maragall Foundation, C/ Wellington 30, 08005 Barcelona, Spain

${ }^{2}$ CIBER Fragilidad y Envejecimiento Saludable (CIBERFES), Madrid, Spain Full list of author information is available at the end of the article
}

and without SCD [3-8]. However, new approaches using more challenging tasks have found subtle deficits in the SCD group [9-12]. Furthermore, the presence of SCD increases the risk of cognitive decline and dementia [7, 13-17] and has been associated with lower volume in the medial temporal lobe, including the hippocampus [18-23], and other AD-related cortical areas [24-26] as compared to cognitively healthy subjects without SCD. Taken together, all of this latter evidence shows that some SCD subjects present structural changes that support the idea of SCD as the first clinical manifestation of AD.

Nevertheless, SCD is a complex syndrome that may be caused by multiple factors besides AD pathology, including other neurological or medical conditions, drug use, or psychological factors (see [27] for a review). Recently, the SCD-Initiative has proposed a set of specific SCD

(c) The Author(s). 2018 Open Access This article is distributed under the terms of the Creative Commons Attribution 4.0 International License (http://creativecommons.org/licenses/by/4.0/), which permits unrestricted use, distribution, and reproduction in any medium, provided you give appropriate credit to the original author(s) and the source, provide a link to the Creative Commons license, and indicate if changes were made. The Creative Commons Public Domain Dedication waiver (http://creativecommons.org/publicdomain/zero/1.0/) applies to the data made available in this article, unless otherwise stated. 
features, under the name SCDplus, which are associated with an increased likelihood to be an expression of the preclinical stage of AD [1,2]. These features are: subjective decline in memory rather than other cognitive domains; onset in the last 5 years; age at onset $>60$ years; concerns (worries) associated with SCD; feeling of worse performance than others of the same age group; confirmation of cognitive decline by an informant; and presence of the $A P O E \& 4$ genotype. There is increasing interest in the study of individuals meeting SCDplus features to assess the usefulness of the SCDplus concept for enriching samples at higher risk of cognitive decline in $\mathrm{AD}$ secondary prevention trials. However, there is limited knowledge on which of the seven proposed features best relates to objective measures of cognition and/or cerebral changes. Most of the studies on SCD have so far included participants attending memory clinics. Recent evidence points out that the clinical, cognitive, and demographic characteristics $[28,29]$ and also the incidence of MCI [30] are different between clinical and population-based SCD samples. The identification of SCDplus cognitive and brain correlates in a population-based cohort would add to the understanding and usefulness of such a concept beyond memory clinics.

In this context, we hypothesize that some differences may be found in SCD subjects from a population-based cohort depending on the number of SCDplus features that they meet. Therefore, the aim of this study is to describe the cognitive and neuroimaging correlates of SCDplus. For this purpose, we describe the cognitive performance in persons with SCD after having classified them as a function of the number of SCDplus features they meet $(\leq 3$ and $>3)$; we investigate whether these features are related to cognitive performance or brain volumes in $\mathrm{AD}$ vulnerable areas (hippocampus); and, finally, we explore brain structural patterns related to the presence of SCDplus features and differences amongst non-SCD and SCD groups using an unbiased voxel-based morphometry (VBM) approach.

\section{Methods}

\section{Participants}

Participants of this study were assessed in the framework of the ALFA project (ClinicalTrials.gov, NCT01835717). ALFA participants $(n=2743)$ are cognitively healthy men and women aged between 45 and 74 years, most of them first-degree descendants of $\mathrm{AD}$ patients $(47.4 \%$ with parental history of $\mathrm{AD}$ with onset before age 75 years). Exclusion criteria included scores below the cutoff values in cognitive screening tests (MMSE $<26$, MIS $<6$, semantic fluency $<12$ ), CDR $>0$, and major psychiatric disorders or diseases that could affect cognitive performance. The complete details on the ALFA study procedures and recruitment have been described in depth elsewhere [31]. For the present study, a total of 2670 subjects have been included since we excluded those individuals from which the $A P O E$ genotype was not available $(n=73)$. The study was approved by the Ethics Committee of the "Parc de Salut Mar" (Barcelona, Spain) and conducted in accordance with the directives of the Spanish Law 14/2007, of 3rd of July, on Biomedical Research. All participants signed an informed consent form and had a close relative, who also granted their consent, volunteering to participate in the study to give information of the participant's cognitive and functional status.

\section{Assessment of subjective cognitive decline and classification of participants}

Participants were classified as SCD if the answer to the question "Do you perceive memory or cognitive difficulties?" was affirmative. To collect standardized information on the perception of cognitive decline we used the Subjective Cognitive Decline Questionnaire (SCD-Q). The SCD-Q was devised to quantify the perceived subjective cognitive decline over the last 2 years and inquiries about the presence or absence of difficulties in 24 cognitive-related activities. The same set of questions is administered to both the subject (SCD-Q MyCog) and the informant (SCD-Q TheirCog) separately [32]. In addition, the presence or absence of six of the seven proposed SCDplus features was determined using either the information gathered by questions in the SCD-Q as proxies (i.e., concern focused in memory, worry about the decline, change in the last 2 years, and confirmation by an informant), sociodemographic data (i.e., age $\geq 60$ years), or genetic testing (i.e., presence of at least one $A P O E \& 4$ allele). Informant confirmation of decline was determined based on the informant answer to the question "Do you perceive he/she has memory or cognitive difficulties?". The SCDplus feature regarding the perception of worse performance than others in the same age group was omitted in the present study, as this information was not collected. The SCD group was dichotomized into two subgroups as a function of the number of SCDplus features that each participant met. The number of features was computed and participants were classified either as SCD minus (SCD-) if they presented $\leq 3$ or as $\mathrm{SCD}$ plus $(\mathrm{SCD}+)$ if they presented $>3$. Participants who did not report SCD (non-SCD) were used as the control sample.

\section{Neuropsychological measures}

The battery used in this study includes measures of verbal episodic memory, executive function and working memory, visual processing, and verbal and nonverbal reasoning. Episodic memory was assessed with the 
Memory Binding Test (MBT), a word list-learning test devised to improve the detection of the earliest memory changes suggestive of AD [33]. The MBT encompasses the sequential learning of two sets of 16 words that share semantic categories. Free, cued, and paired recall are assessed in immediate and delayed (after 25-35 min) trials. We used the Spanish version of the MBT that we have recently adapted, normed, and validated [34]. The following main variables were gathered and analyzed: immediate total paired recall (TPR), immediate total free recall (TFR), total delayed paired recall (TDPR), and total delayed free recall (TDFR). The Wechsler IV Coding, Digit Span, Visual Puzzles, Similarities, and Matrix Reasoning subtests were also administered [35]. The coding task measures, among others, processing speed and attention. The Digit Span subtest evaluates short-term and working memory. Visual Puzzles measures visual reasoning and processing. Matrix Reasoning assesses fluid intelligence, and Similarities measures verbal reasoning and abstract thinking.

\section{Anxiety and depression}

The Goldberg Anxiety and Depression Scale (GADS) was administered to assess the presence of anxiety and depressive symptoms. The scale is composed of two subscales with a maximum score of 9 points each. A global score is computed by summing up both subscales [36].

\section{MRI acquisition and preprocessing}

A subgroup of 532 participants underwent a structural magnetic resonance imaging (MRI) study. This subgroup was selected as a function of their $A P O E$ genotype, aiming to maximize the number of $A P O E \& 4$ allele carriers, which is known to be the most relevant genetic risk factor for AD [37]. Three-dimensional high-resolution T1-weighted images were obtained using a $3 \mathrm{~T}$ General Electric Discovery scanner with the following acquisition parameters: fast spoiled gradient-echo sequence, voxel size $=1 \mathrm{~mm}^{3}$ isotropic, repetition time $(\mathrm{TR})=6.16 \mathrm{~ms}$, echo time $(\mathrm{TE})=2.33 \mathrm{~ms}$, inversion time $(\mathrm{TI})=450 \mathrm{~ms}$, matrix size $=256 \times 256 \times 174$, flip angle $=12^{\circ}$. Images were processed to perform voxel-wise statistics with the following procedure. They were segmented into gray matter (GM) tissue using the new segment function implemented in Statistical Parametrical Mapping software (SPM 12; Wellcome Department of Imaging Neuroscience, London, UK). The DARTEL toolbox was used to generate a reference template object of the sample which was warped into a standard MNI space. The generated flow fields and normalization parameters were then implemented to normalize the native GM T1 images to the MNI space. Jacobian determinants were applied to preserve the local native amount of gray matter (modulated images). Finally, images were spatially smoothed with a 10-mm full-width at half maximum (FWHM) Gaussian kernel. Additionally, the total intracranial volume (TIV) was computed by summing the segmented GM, WM, and CSF for each individual. Hippocampal volumes were automatically calculated by FreeSurfer (v5.3; surfer.nmr.mgh.harvard.edu) using whole hippocampal segmentation provided by the standard 'recon-all' pipeline, which renders the most standard and widely-used volumetric estimation of hippocampal volumes. Additional details on the computation of hippocampal volumes by this pipeline can be found in [38]. Raw hippocampal volumes were adjusted by dividing them by the TIV.

\section{Statistical analyses}

We compared the means in sociodemographic and cognitive screening variables as well as $A P O E \& 4$ allele frequency distributions between non-SCD subjects and the whole SCD group by means of $t$ tests for continuous variables and chi-square tests for categorical variables. After splitting the SCD group into two subgroups as a function of the number of SCDplus features they meet ( $\mathrm{SCD}-$ and $\mathrm{SCD}+$ ), these variables were compared by one-way ANOVA and pairwise post-hoc tests (Tukey) and chi-square tests. Similarly, cognitive performance was firstly compared between non-SCD subjects and the SCD group as a whole and, secondly, among non-SCD subjects and SCD subgroups using ANCOVAs (with age, years of education, sex, number of $A P O E \varepsilon 4$ alleles, and mood scores as covariates). In this second analysis, both the main effect of group (non-SCD/SCD-/SCD+) and post-hoc pairwise differences were tested. TIV-adjusted hippocampal volumes were first compared among SCD groups by mean ANOVAs and subsequently using covariation by age, sex, and number of $A P O E \& 4$ alleles using ANCOVAs. In an additional analysis, the association between the independent SCDplus features and cognitive performance was tested with linear models in which SCDplus features were included as predictor variables, cognitive performance as dependent variables, and age, education, sex, and mood scores as covariates. Similarly, models with the TIV-adjusted hippocampal volume as the dependent variable were constructed to explore its association with the SCDplus features. Statistical analysis was performed in SPSS IBM v22. All tests were two-tailed, with a significant level of $\alpha=0.05$.

Voxel-wise analyses were performed using the general linear model as implemented in SPM 12. The segmented, modulated, and smoothed GM images were entered in a full-factorial design and differences in GM volume were explored using pairwise contrasts among the non-SCD, SCD-, and SCD+ groups. Age, education, sex, mood scores, and TIV were used as nuisance variables. In addition, we introduced the number of $A P O E$ $\varepsilon 4$ alleles as a covariate to regress its effect out of our 
VBM models under the assumption that the effect of the number of alleles is additive [39]. Additional models were constructed to explore the association between SCDplus features and GM volumes. Firstly, we tested the association between the number of SCDplus features met and GM volume using covariation by age, education, sex, and TIV. Secondly, a model was built including the six binary individual features as effects of interest and age, education, sex, and TIV as covariates. Contrasts for each feature were tested separately. An uncorrected $p$ value $(<0.001)$ and a minimum cluster size $(k=100)$ were used as significance thresholds in all VBM analyses performed due to the exploratory nature of the study assuming that few differences between these groups of healthy participants would be observed. Family-wise error (FWE)-corrected $p$ values were also computed and provided for reference.

\section{Results}

\section{Prevalence of SCD and SCDplus features}

Among the ALFA participants included $(n=2670), 21.4 \%$ ( $n$ $=572$ ) accomplished criteria for SCD. With regard to SCDplus features, all reported memory as the main compromised domain, $72 \%$ experienced the decline within the last 2 years, $67.8 \%$ expressed worries about their cognition, $34.3 \%$ were $A P O E \& 4$ carriers, $34.1 \%$ were older than 60 years, and, finally, $28 \%$ had confirmation of decline from an informant. Prevalence of features by SCD subgroups (SCD+ and SCD-) are presented in Table 1 . See Additional file 1 for a more detailed description of frequency and co-occurrence of SCDplus features (Additional file 1: Tables S2 and S3 and Figures S1 and S2).
As a whole group, SCD individuals were older and reported higher anxiety and depressive symptoms than participants not presenting SCD $(p<0.001)$. Table 1 presents descriptive data for sociodemographic information, the number of $A P O E \& 4$ carriers, and mood scores by $\mathrm{SCD}$ categories. As expected, SCD+ individuals were older and had a higher prevalence of the $A P O E \varepsilon 4$ allele than the non-SCD and SCD- groups $(p<0.001)$. We did not find statistically significant differences in the anxiety and depression subscales of the GADS between the SCD + and SCD- subgroups. Regarding SCD-Q scores, SCD+ subjects obtained higher scores than the SCD- and non-SCD subjects $(p<0.001)$. This held true for both the participant scores $(\mathrm{MyCog})$ and the informant scores (TheirCog). Noteworthy, self-reported difficulties (assessed as the overall MyCog score) in all groups were higher than informant-derived reports (TheirCog overall score) with increasing values following the pattern non-SCD $<$ SCD $-<$ SCD + .

The subsample of subjects who underwent MRI was on average 1 year younger $(p<0.05)$ and slightly more educated $(0.3$ years of education, $p<0.05)$ than the group that was not scanned. Sociodemographic, genetic, and mood data of this subgroup are presented in Additional file 1: Table S1. The percentage of APOE $\varepsilon 4$ allele carriers is overrepresented in the MRI subsample $(30.7 \%$ vs $51 \%, p<0.001)$. No significant differences in mood or cognitive outcomes were found, except a marginally better performance of scanned individuals in the Similarities subtest $(p<0.05)$. With regard to SCD prevalence, it was similar in both groups $(21.7 \%$ vs $20.5 \%$ in the MRI subsample, $p<0.54)$. Noticeably, the number of subjects

Table 1 Sociodemographic, genetic, mood, and SCDplus features descriptive data in the whole sample

\begin{tabular}{|c|c|c|c|}
\hline & Non-SCD group & SCD- group & $\mathrm{SCD}+$ group \\
\hline $\bar{N}$ & 2098 & 319 & 253 \\
\hline Age, mean (SD) & $55.41(6.62)$ & $55.62(6.22)$ & $59.10(7.12)^{*, * *}$ \\
\hline Education, mean (SD) & $13.41(3.52)$ & $13.38(3.55)$ & $12.83(3.41)^{*}$ \\
\hline Females, $n(\%)$ & $1316(62.7 \%)$ & $218(68.3 \%)$ & $154(60.9 \%)$ \\
\hline GADS—anxiety, mean (SD) & $0.49(1.07)$ & $0.92(1.51)^{*}$ & $0.90(1.41)^{*}$ \\
\hline GADS—depression, mean (SD) & $0.11(0.52)$ & $0.35(0.93)^{*}$ & $0.44(1.07)^{*}$ \\
\hline \multicolumn{4}{|l|}{ SCDplus features, $n(\%)$} \\
\hline Worry about the decline & & $165(51.7 \%)$ & $223(88.1 \%)^{* *}$ \\
\hline Change in the last 2 years & & $179(56.1 \%)$ & $233(92.1 \%)^{* *}$ \\
\hline Age $>60$ years & & $62(19.4 \%)$ & $133(52.6 \%)^{* *}$ \\
\hline Confirmation by informant $^{a}$ & & $37(11.6 \%)$ & $122(48.2 \%)^{* *}$ \\
\hline$A P O E \& 4$ carriers & $731(34.8 \%)$ & $52(16.3 \%)^{*}$ & $144(56.9 \%)^{* * * *}$ \\
\hline
\end{tabular}

SCDplus subjective cognitive decline plus, non-SCD not self-reporting cognitive decline, $S C D-S C D$ with $\leq 3$ SCDplus features, $S C D+S C D$ with $>3$ SCDplus features, $S C D$ subjective cognitive decline, SD standard deviation, GADS Goldberg Anxiety and Depression Scale, APOE apolipoprotein E, ANOVA analysis of variance

${ }^{\text {a }}$ Data available in 568 out of 572 participants with SCD

${ }^{*} p<0.05$ as compared to non-SCD; pairwise (Tukey) ANOVA/chi-square test

*** $p<0.05$ as compared to SCD-; pairwise (Tukey) ANOVA/chi-square test 
classified as $\mathrm{SCD}+$ was higher in the MRI subsample (44.2\% vs $66.1 \%, p<0.05)$.

\section{Cognitive outcomes}

Participants with SCD obtained lower scores than non-SCD subjects in all memory variables $(p<0.05)$. However, we found that these differences were mainly driven by the performance of the SCD+ group, because this group presented statistically significant differences with respect to the non-SCD group, while the SCDgroup did not (Table 2). The SCD+ group also displayed significantly lower scores than non-SCD participants in Coding. This difference survived a Bonferroni-type correction for multiple comparisons using a more restrictive $p$ value $(p<0.005)$, as did the MBT-TDFR variable, in which the $\mathrm{SCD}+$ group showed significant differences as compared to both the SCD- group and the non-SCD group. Figure 1 provides a graphic example for the MBT-TDFR. Although differences were statistically significant, the magnitudes of the effects were small (largest partial $\eta^{2}=0.009$ ).

\section{Neuroimaging results for group comparisons}

The SCD+ group showed lower left TIV-adjusted hippocampal volumes than the non-SCD $(p=0.004)$ and SCD- $(p=0.017)$ groups in unadjusted comparisons. However, after covariation by age, sex, and number of $A P O E \& 4$ alleles this difference was no longer significant $(p<0.05)$. In the VBM analysis, the SCD+ group showed significantly lower GM volume than the SCD- group in regions known to be affected in $\mathrm{AD}$ such as the bilateral temporal cortices, fusiform and lingual gyri, precuneus and cuneus, and medial cerebellum (Fig. 2a and Table 3). No significant differences were detected between the $\mathrm{SCD}+$ and non-SCD groups. On the other hand, the $\mathrm{SCD}$ - group showed increased GM volume with respect to the non-SCD group in a very similar pattern as observed in the comparison between the SCD- and SCD+ groups (Fig. 2b and Table 3).

\section{Effect of SCDplus features in cognition and neuroimaging outcomes}

We found that confirmation of cognitive decline by an informant predicted the MBT immediate and delayed total free recalls (TFR and TDFR) (TFR, standardized $\beta$ $=-0.31, p=0.046$; TDFR, standardized $\beta=-0.37, p=$ $0.004)$. In addition, both confirmation by an informant and age $>60$ years predicted performance in the Coding subtest (confirmation by informant, standardized $\beta=-$ $0.24, p=0.046$; age $>60$ years, standardized $\beta=-0.33, p$ $=0.005$ ). Furthermore, we found a positive effect in the performance on Matrix Reasoning for being worried about the decline (standardized $\beta=0.17, p=0.025$ ). No other features significantly predicted performance in the cognitive outcomes assessed here. Setting a more restrictive $p$ value to adjust for multiple comparisons $(p>$ 0.005), the relationship between TDFR and confirmation of decline and the relationship between age $>60$ years and Coding were still significant.

With regard to neuroimaging outcomes, confirmation of the cognitive decline by an informant was the only SCDplus feature that predicted lower hippocampal

Table 2 SCD-Q and cognitive scores by SCD group

\begin{tabular}{llll}
\hline & Non-SCD group & SCD-group & SCD+ group \\
\hline N & 2098 & 319 & 253 \\
SCD-Q MyCog & $4.35(4.06)$ & $10.39(5.66)^{*}$ & $14.04(5.41)^{* * * * *}$ \\
SCD-Q TheirCog & $2.78(2.44)$ & $4.02(4.03)^{*}$ & $6.39(5.25)^{* * * *}$ \\
MBT-TPR & $24.29(4.26)$ & $23.89(4.48)$ & $22.52(4.98)^{* * * *}$ \\
MBT-TFR & $16.74(4.93)$ & $16.36(4.90)$ & $14.51(5.11)^{* * * *}$ \\
MBT-TDFR & $17.14(3.02)$ & $16.68(5.42)$ & $14.67(5.23)^{* * * *}$ \\
MBT-TDPR & $24.11(4.41)$ & $23.75(4.62)$ & $22.23(5.17)^{* * * *}$ \\
WAIS-Coding & $66.67(14.6)$ & $65.63(14.91)$ & $59.64(13.90)^{*}$ \\
WAIS-Visual Puzzles & $13.55(4.30)$ & $12.90(4.12)$ & $12.26(3.90)$ \\
WAIS-Digit Span Total & $24.93(5.11)$ & $24.66(5.33)$ & $23.86(5.58)$ \\
WAIS-Matrix Reasoning & $16.77(4.35)$ & $16.31(4.51)$ & $15.41(4.48)$ \\
WAIS-Similarities & $22.34(4.64)$ & $21.67(4.80)$ & $21.74(4.73)$ \\
\hline
\end{tabular}

Data presented as mean (standard deviation)

$S C D-Q$ Subjective Cognitive Decline Questionnaire, $S C D$ subjective cognitive decline, non-SCD not self-reporting cognitive decline, $S C D$ - SCD with $\leq 3$ SCDplus features, SCD+ SCD with > 3 SCDplus features, SCDplus subjective cognitive decline plus, MyCog questions to participant, TheirCog questions to informant, MBT Memory Binding Test, TPR total paired recall, TFR total free recall, TDFR total delayed free recall, TDPR total delayed paired recall, WAIS Wechsler Adult Intelligence Scale, $A P O E$ apolipoprotein $\mathrm{E}$

${ }^{*} p<0.05$ as compared to non-SCD

${ }^{* *} p<0.05$ as compared to SCD-. Analysis of variance and post-hoc Tukey test for SCD-Q; pairwise (Least Significant Difference) analysis of covariance with age, education, sex, number of $A P O E \varepsilon 4$ alleles, anxiety, and depression as covariates for cognitive outcomes 
A

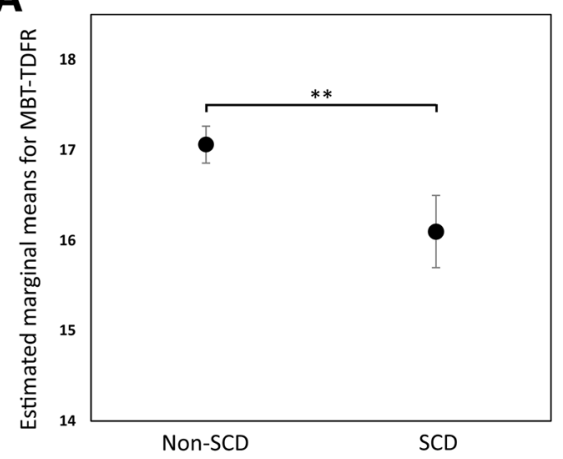

B

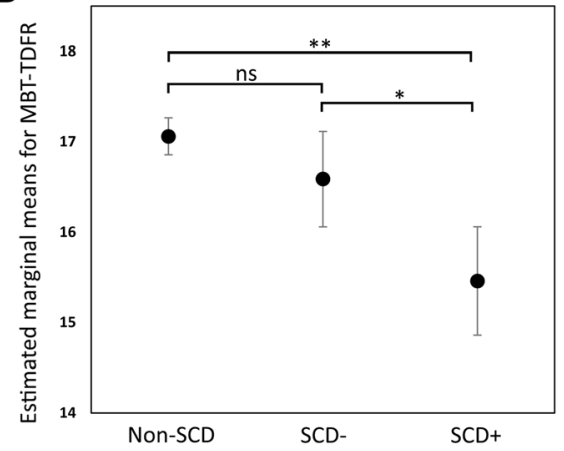

Fig. 1 Estimated marginal means for total delayed free recall (TDFR) of the Memory Binding Test (MBT) by SCD group. a Non-SCD vs SCD. b Non-SCD vs SCD subgroups by number of SCDplus features met (SCD+, SCD-). ${ }^{*} p<0.005$, ${ }^{*} p<0.0001$, ns not significant, SCD self-reporting cognitive decline, non-SCD not self-reporting cognitive decline, SCD + SCD with $>3$ subjective cognitive decline plus features, SCD- SCD with $\leq 3$ subjective cognitive decline plus features

volume (left hippocampus, standardized $\beta=-0.20, p=$ 0.033 ; right hippocampus, standardized $\beta=-0.21, p=$ 0.046). In a posterior analysis, we explored the correlation between hippocampal volume and episodic memory performance (TFR and TDFR) accounting for the presence or absence of informant confirmation of decline. A positive correlation with TDFR was found in SCD subjects who had informant confirmation $(n=39)$, although no significance was reached (left hippocampus, $r=0.20, p=0.22$; right hippocampus, $r=0.25, p=0.12$; Fig. 3). This finding was confirmed by the results obtained from the VBM analysis. Confirmation of the decline by the informant was the feature that more consistently showed association with lower GM volume in AD-related areas, encompassing the bilateral inferior temporal lobe, left hippocampus, right insula, and right orbitofrontal cortex (Fig. 4). The SCDplus feature of being older than 60 years was associated with lower GM volume in the right supramarginal gyrus ( $>60$ years), while reporting worries about the decline was associated with lower GM in the right superior temporal gyrus and superior parietal, and left insula. We found a

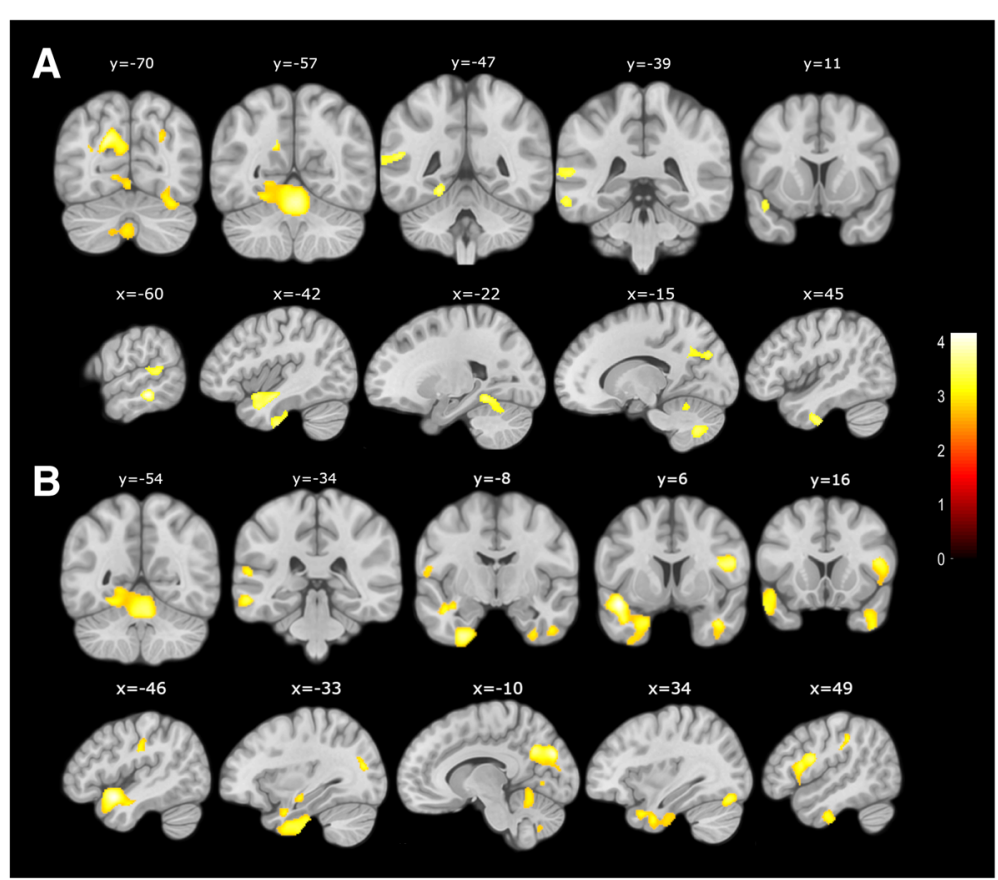

Fig. 2 SPM maps showing contrasts which showed significant differences at $p<0.001$ and minimum cluster size $k=100$. a Areas of decreased gray matter volume of SCD+ subjects as compared to SCD- subjects (SCD- > SCD+). $\mathbf{b}$ Areas of increased gray matter volume of SCD- subjects as compared to non-SCD subjects (SCD- > non-SCD) 
Table 3 Brain regions showing statistically significant gray matter volumetric decrease in pairwise comparisons between non-SCD, SCD+, and SCD- groups

\begin{tabular}{|c|c|c|c|c|c|c|c|c|c|}
\hline \multirow[t]{2}{*}{ Contrast } & & \multirow[t]{2}{*}{ Label } & \multicolumn{3}{|c|}{ MNI coordinates } & \multirow{2}{*}{$\begin{array}{l}\text { Cluster } \\
\text { size }\end{array}$} & \multirow{2}{*}{$\begin{array}{l}\text { Peak } \\
Z \text { score }\end{array}$} & \multicolumn{2}{|c|}{ pFWE-corr } \\
\hline & & & $x$ & $y$ & $z$ & & & Peak & Cluster \\
\hline \multirow[t]{27}{*}{ SCD- > SCD+ } & Cluster 1 & Left temporal middle & -59 & -33 & -12 & 345 & 4.13 & 0.117 & 0.459 \\
\hline & & Left temporal inferior & & & & & & & \\
\hline & Cluster 2 & Left temporal inferior & -33 & -15 & -44 & 801 & 4.05 & 0.151 & 0.164 \\
\hline & & Left fusiform & & & & & & & \\
\hline & Cluster 3 & Left temporal pole superior & -38 & -11 & -17 & 753 & 3.81 & 0.315 & 0.182 \\
\hline & & Left temporal middle & & & & & & & \\
\hline & & Left temporal superior & & & & & & & \\
\hline & & Left temporal pole middle & & & & & & & \\
\hline & Cluster 4 & Right cerebellum & 30 & -74 & -20 & 734 & 3.73 & 0.393 & 0.190 \\
\hline & & Right Fusiform & & & & & & & \\
\hline & Cluster 5 & Left postcentral & -53 & -20 & 30 & 208 & 3.72 & 0.401 & 0.625 \\
\hline & & Left supramarginal & & & & & & & \\
\hline & Cluster 7 & Right cerebellum & 12 & -66 & -47 & 556 & 3.72 & 0.403 & 0.283 \\
\hline & & Vermis & & & & & & & \\
\hline & Cluster 8 & Vermis & -2 & -59 & -18 & 1540 & 3.68 & 0.437 & 0.037 \\
\hline & & Left cerebellum & & & & & & & \\
\hline & & Left fusiform & & & & & & & \\
\hline & & Right cerebellum & & & & & & & \\
\hline & Cluster 9 & Left cerebellum & -14 & -68 & -45 & 559 & 3.63 & 0.494 & 0.281 \\
\hline & Cluster 10 & Left occipital superior & -17 & -77 & 29 & 340 & 3.59 & 0.536 & 0.464 \\
\hline & & Left precuneus & & & & & & & \\
\hline & & Left cuneus & & & & & & & \\
\hline & Cluster 11 & Right temporal inferior & 47 & -9 & -38 & 420 & 3.59 & 0.541 & 0.386 \\
\hline & & Right temporal pole & & & & & & & \\
\hline & & Right fusiform & & & & & & & \\
\hline & Cluster 12 & Left temporal superior & -54 & -45 & 15 & 428 & 3.54 & 0.593 & 0.379 \\
\hline & & Left temporal middle & & & & & & & \\
\hline \multirow[t]{15}{*}{ SCD- > non-SCD } & Cluster 1 & Left temporal pole superior & -45 & 6 & -17 & 4502 & 4.66 & 0.015 & 0.000 \\
\hline & & Left fusiform & & & & & & & \\
\hline & & Left temporal pole middle & & & & & & & \\
\hline & & Left temporal superior & & & & & & & \\
\hline & & Left hippocampus & & & & & & & \\
\hline & & Left parahippocampal & & & & & & & \\
\hline & & Left insula & & & & & & & \\
\hline & & Left amygdala & & & & & & & \\
\hline & Cluster 2 & Left cuneus & -17 & -63 & 33 & 2216 & 4.56 & 0.022 & 0.011 \\
\hline & & Left occipital superior & & & & & & & \\
\hline & & Left precuneus & & & & & & & \\
\hline & & Right cuneus & & & & & & & \\
\hline & & Left calcarine & & & & & & & \\
\hline & Cluster 3 & Right fusiform & 30 & -77 & -17 & 956 & 4.31 & 0.061 & 0.118 \\
\hline & & Right cerebellum & & & & & & & \\
\hline
\end{tabular}


Table 3 Brain regions showing statistically significant gray matter volumetric decrease in pairwise comparisons between non-SCD, SCD+, and SCD- groups (Continued)

\begin{tabular}{|c|c|c|c|c|c|c|c|c|c|}
\hline \multirow[t]{2}{*}{ Contrast } & & \multirow[t]{2}{*}{ Label } & \multicolumn{3}{|c|}{ MNI coordinates } & \multirow{2}{*}{$\begin{array}{l}\text { Cluster } \\
\text { size }\end{array}$} & \multirow{2}{*}{$\begin{array}{l}\text { Peak } \\
Z \text { score }\end{array}$} & \multicolumn{2}{|c|}{ pFWE-corr } \\
\hline & & & $x$ & $y$ & $z$ & & & Peak & Cluster \\
\hline & & Right lingual & & & & & & & \\
\hline & & Right occipital inferior & & & & & & & \\
\hline & Cluster 4 & Left lingual & 2 & -57 & -18 & 3020 & 4.28 & 0.068 & 0.003 \\
\hline & & Vermis & & & & & & & \\
\hline & & Left cerebellum & & & & & & & \\
\hline & & Left fusiform & & & & & & & \\
\hline & Cluster 5 & Right temporal pole middle & 39 & 9 & -33 & 1723 & 4.15 & 0.109 & 0.026 \\
\hline & & Right temporal inferior & & & & & & & \\
\hline & & Right temporal pole superior & & & & & & & \\
\hline & Cluster 6 & Right frontal inferior & 50 & 2 & 20 & 1157 & 4.11 & 0.126 & 0.078 \\
\hline & & Right rolandic & & & & & & & \\
\hline & & Right precentral & & & & & & & \\
\hline & Cluster 7 & Left temporal middle & -59 & -33 & -12 & 317 & 3.99 & 0.183 & 0.489 \\
\hline & & Left temporal inferior & & & & & & & \\
\hline & Cluster 8 & Left occipital middle & -32 & -75 & 15 & 123 & 3.93 & 0.220 & 0.749 \\
\hline & Cluster 9 & Right supramarginal & 45 & -30 & 33 & 189 & 3.72 & 0.404 & 0.651 \\
\hline & & Right postcentral & & & & & & & \\
\hline & Cluster 10 & Left temporal superior & -59 & -35 & 12 & 250 & 3.58 & 0.551 & 0.569 \\
\hline & & Left temporal middle & & & & & & & \\
\hline & Cluster 11 & Left postcentral & -53 & -17 & 32 & 396 & 3.58 & 0.554 & 0.408 \\
\hline & & Left parietal inferior & & & & & & & \\
\hline & & Left supramarginal & & & & & & & \\
\hline & Cluster 12 & Right cerebellum & -3 & -68 & -45 & 489 & 3.56 & 0.601 & 0.330 \\
\hline & & Left cerebellum & & & & & & & \\
\hline & Cluster 13 & Left rolandic & -60 & -3 & 12 & 229 & 3.53 & 0.624 & 0.596 \\
\hline & & Left postcentral & & & & & & & \\
\hline & Cluster 14 & Right occipital superior & 26 & -71 & 33 & 109 & 3.40 & 0.617 & 0.771 \\
\hline
\end{tabular}

non-SCD not self-reporting cognitive decline, $S C D-S C D$ with $\leq 3$ SCDplus features, $S C D+S C D$ with $>3$ SCDplus features, $S C D p l u s$ subjective cognitive decline plus, MNI Montreal Neurological Institute, pFWE Family-wise Error-adjusted $p$-value

negative association between the number of SCDplus features met by SCD participants and GM local volumes showing a topographical distribution that included areas in the temporal lobe and precuneus bilaterally, the cerebellum, and some frontal regions (see Additional file 1: Figure S3 and Table S4).

\section{Discussion}

In this study, performed in a large community-based sample of middle-aged healthy subjects, we aimed to determine whether meeting more than three SCDplus features had specific cognitive and neuroimaging correlates, and also to explore which features better predicted cognitive performance and GM volume. Overall, our findings support the use of the concept of SCDplus also in population-based cohorts since performance in subjective decliners was lower than that in non-SCD participants only in the individuals meeting more than three SCDplus features ( $\mathrm{SCD}+$ group). This group also showed a decrease of volume in $\mathrm{AD}$-related brain areas as compared to the SCD group meeting fewer features. We found that confirmation of decline by an informant was the best cognitive and GM volume predictor.

In this population-based cohort, 572 out of the 2670 subjects studied reported SCD, representing a prevalence of $21.4 \%$. SCD prevalence is highly variable among studies, depending upon sample recruitment strategies as well as the SCD definition [40], our findings being in accordance with previous community-dwelling SCD studies $[41,42]$. As compared to non-SCD participants, subjects with SCD reported higher anxiety and depressive symptoms and had higher scores on the SCD-Q 

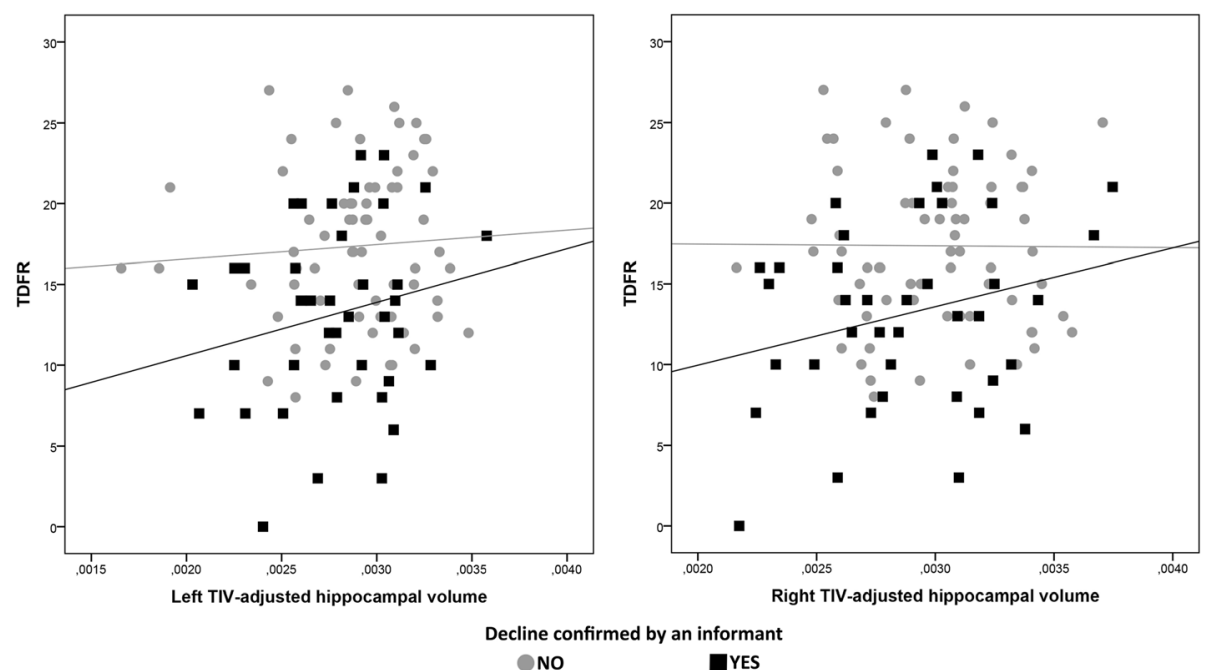

Fig. 3 Association between memory scores and TIV-adjusted hippocampal volumes by presence/absence of informant confirmation of decline. TDFR total delayed free recall, TIV total intracraneal volume

questionnaire. Besides the compromise in memory, the most prevalent SCDplus features were a reported decline in the last 2 years and the presence of worries about the decline.

We found a significant negative impact of SCD in cognition, mainly driven by the SCD+ group (individuals meeting $>3$ SCDplus features). A significant lower performance in this group was observed as compared to both non-SCD subjects and subjects in the SCD- group, while no differences between these latter groups were found. These findings could not be attributable to confounders, since adjustments for demographics and mood scores were implemented in the analysis. Differences were focused in the episodic memory domain, which mainly relies on the integrity of medial temporal lobe regions [43]. There is a well-described spread of neuropathology from these regions to wide cortical areas in the course of symptomatic $\mathrm{AD}$, symptoms being related to the topographical distribution of tau pathology. Recently, evidence of increased tau deposition has been reported in the medial temporal lobe of healthy elders with SCD [44], and our findings on the predominance of memory subclinical deficits in subjects with SCD may be related to such changes.

Our results for cognitive outcomes partially mirror the findings reported by Fernández-Blázquez et al. in subjects who fulfill SCDplus features [45]. They found lower scores in SCDplus as compared to non-SCD subjects only for verbal episodic memory measured with the Free and Cued Selective Reminding Test (FCSRT). Interestingly, in their study they found differences between SCDplus and SCD restricted to the delayed free recall scores. In our study we used the MBT, which is a test that has similar advantages to the FCSRT (i.e., controlled learning and distinction

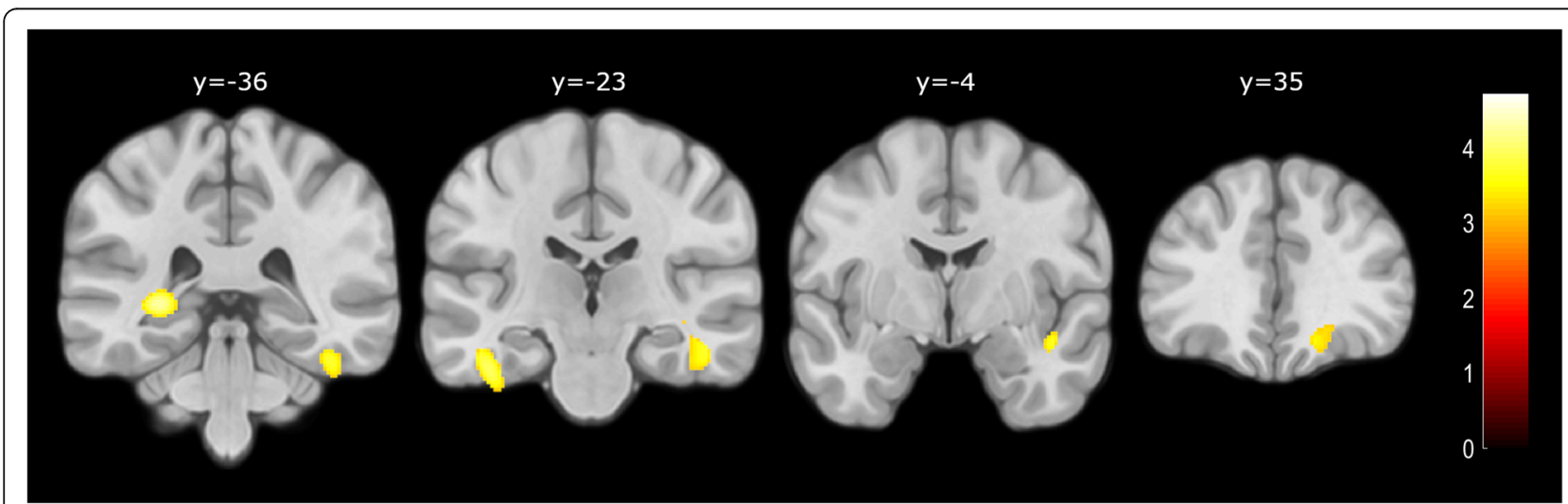

Fig. 4 Areas in which confirmation of cognitive decline by an informant is associated with decreased gray matter volume 
between storage and retrieval) but overcomes its limitations, such as the ceiling scores achieved by subjects with any or subtle memory deficits [46]. By using a larger sample and the MBT, we were able to demonstrate global memory differences, in both free and cued measures, between the SCD+ and SCD- groups.

We also found differences in the Coding subtest of the WAIS-IV. This kind of task, which measures attention and psychomotor and processing speed, is one of the most sensitive tools to detect cognitive impact due to any brain insult but lacks disease-related specificity [47]. Noteworthy, this test was included in the longitudinal data-derived preclinical Alzheimer cognitive composite (PACC) and, together with episodic memory and orientation measures, has shown good performance in detecting decline in cognitively healthy subjects with evidence of AD pathology [48].

With regard to the ability of specific SCDplus features to predict cognitive performance, in our study, confirmation of the decline by an informant was the feature that best related to objective cognitive performance in memory and executive function tasks. In addition, this feature was also the only one that predicted hippocampal volume and related to lower GM volume in AD-relevant regions. This is in line with several reports highlighting the value of informant ratings in SCD and preclinical $\mathrm{AD}$ in which informant complaints predicted progression to AD dementia in longitudinal studies $[49,50]$. Recently, Valech et al. [51] demonstrated the superiority of informant-related ratings over self-reported ones when it comes to discriminating between controls and biomarker-defined preclinical AD subjects. In addition, they found that informant measures also correlated better with CSF AD biomarkers than self-derived measures. The rationale behind this phenomenon is proposed to be two-fold: first, some subjects in the AD preclinical stage may present some initial form of anosognosia (unawareness of the own difficulties); and second, a proportion of the cognitive decliners report subjective changes related to other conditions, such as psychoaffective symptoms, or personality traits, such as neuroticism. In this regard, in our study, subjects with SCD reported higher anxiety and depressive symptoms as compared to non-SCD subjects, as previously reported [28, 52]. This finding is in agreement with the attentional bias toward negative information that depressed individuals usually present [53], which would make them more sensitive to their cognitive failures [54] and points toward an overlap between the subjective perception of decline due to preclinical AD and due to mood-related causes. In both scenarios, anosognosia and mood-driven complaints, external reports appear as less biased indicators of the presence of actual subclinical decline. Investigators in the INSIGHT-preAD study have further explored this by developing an "awareness indicator", operationalized as the difference between informant-reported and self-reported scores. This type of measure seems promising since, by classifying subjects in "low versus high awareness" groups, significant differences that were not captured by isolated SCD scores appear in amyloidosis and reduced cortical metabolism [4].

With regard to other SCDplus features, we found that being older than 60 years, even after age adjustment, predicted lower performance in Coding. This finding suggests higher executive/processing speed impact of SCD than that expected by age. In contrast, we found a positive relationship between having worries about the decline and Matrix Reasoning performance. An association between higher insight and good reasoning abilities could underlie this unexpected association.

As expected, the $\mathrm{SCD}+$ group showed lower GM volume than the SCD- one in areas known to be affected by $\mathrm{AD}$. On the other hand, we found that the non-SCD group showed no significant differences with respect to the $\mathrm{SCD}+$ group, and also displayed lower GM volumes than the SCD- group. This effect overlapped with those regions with decreased $\mathrm{GM}$ volume in the $\mathrm{SCD}+$ group as compared to the SCD- group. Indeed, in an independent population composed of subjects in the $\mathrm{AD}$ continuum from healthy controls to preclinical and symptomatic $\mathrm{AD}$, we previously reported a nonlinear association pattern between brain volumes and CSF biomarker levels in some brain regions [55]. In this previous study, the parahippocampus and some parietotemporal regions presented an initial increase in volume in the low to intermediate biomarker abnormality level that later reverted. In this context, we hypothesize that the observed increase in GM volumes found in the SCD- group presented here might be capturing this initial effect of AD pathology that may be present in some subjects. Within the SCD group, on the contrary, the SCDplus classification criteria showed the capacity to detect individuals who, on average, displayed brain morphological differences associated with preclinical $A D$ stages. In this regard, note that our sample was recruited from the general population rather than from memory clinics as in most SCD studies found in the literature. Therefore, the particular recruitment strategy in our study may underlie this unexpected finding which deserves further investigation. It is known that the recruitment strategy could highly affect SCD characteristics. Perrotin et al. [28] found differences between SCD subjects recruited from a memory clinic and those from the community. While both groups showed increased $\beta$-amyloid deposition and anxiety, subclinical depression and brain atrophy was found only in the SCD subjects who sought medical advice. Using VBM they reported brain atrophy in similar areas to those we found in our study in this latter group, suggesting that the presence of 
worry of enough intensity to induce help-seeking behavior would be a relevant feature of increased likelihood for AD. Similarly, Abdelnour et al. [29] reported worse neuropsychological performance in individuals with SCD attending a memory clinic than those recruited as study participants from the community, which may reflect a higher enrichment of $\mathrm{AD}$ in this group. Help-seeking has been recently suggested to occur concurrent with NIA-AA preclinical stage 2 (i.e., evidence of positive amyloid and tau markers) and to be strongly related to future cognitive decline $[17,30]$. In the whole picture, our findings in the ALFA population-based cohort may reflect changes at an earlier stage to help-seeking behavior that can be captured at the group level by neuroimaging and challenging cognitive assessments.

This study is not free of limitations. The most important is the absence of biomarker data to be used as proxies of AD pathology. Ongoing studies in the follow-up of a subsample will solve this and will enable a refined analysis to further assess the usefulness of using the number of SCDplus features as a proxy of a continuum within SCD. In addition, as already highlighted throughout this paper, the value of each individual SCDplus feature seems to have a different weight in the prediction of cognitive performance. Ideally, homogeneous subgroups of subjects fulfilling each of the possible feature combinations would aid to disentangle interactions and reveal combinations of features of special interest. However, the sample size and the frequency of SCDplus features in the current study prevented us from using this approach.

\section{Conclusions}

This study provides evidence of distinct cognitive and neuroimaging correlates of SCDplus subjects. Our analysis revealed complex interplays between cognitive performance and brain morphometry profiles that deserve further investigation. This approach may contribute to developing strategies to stratify SCD subjects at different risk levels of AD.

\section{Additional file}

Additional file 1: Table S1. Sociodemographic, genetic, and mood descriptive data in the MRI sample. Table S2. Frequency of co-occurrence of SCDplus features in subjects with SCD in the whole sample $(n=572)$. Table S3. Frequency of co-occurrence of SCDplus features in subjects with SCD in the MRI subsample $(n=109)$. Table S4. Brain regions showing statistically significant negative associations between the number of SCDplus features met and $\mathrm{GM}$ volume. Figure S1. Frequency of SCD subjects by number of criteria met in the whole sample. Figure $\mathbf{S 2}$. Frequency of SCD subjects by number of criteria met in the MRI subsample. Figure S3. Brain regions showing a negative linear relation between number of SCDplus features met and GM volume in SCD subjects. (DOCX $441 \mathrm{~kb}$ )

\section{Abbreviations}

AD: Alzheimer's disease; APOE: Apolipoprotein E; CDR: Clinical Dementia Rating; CSF: Cerebrospinal fluid; FCSRT: Free and Cued Selective Reminding Test; FWHM: Full-width at half maximum; GADS: Goldberg Anxiety and
Depression Scale; GM: Gray matter; MBT: Memory Binding Test; MCI: Mild cognitive impairment; MIS: Memory Impairment Screen; MMSE: Mini-Mental State Examination; MNI: Montreal Neurological Institute; MRI: Magnetic resonance imaging; PACC: Preclinical Alzheimer cognitive composite; SCD: Subjective cognitive decline; SCD-Q: Subjective Cognitive Decline Questionnaire; TDFR: Total delayed free recall; TDPR: Total delayed paired recall; TFR: Total free recall; TIV: Total intracraneal volume; TPR: Total paired recall; VBM: Voxel-based morphometry; WAIS: Wechsler Adult Intelligence Scale; WM: White matter

\section{Acknowledgements}

This publication is part of the ALFA study (ALzheimer and FAmilies). The authors would like to express their most sincere gratitude to the ALFA project participants, without whom this research would have not been possible.

Collaborators of the ALFA study are: Jordi Camí, Grégory Operto, Stavros Skouras, Karine Fauria, Anna Brugulat-Serrat, Gemma Salvadó, Albina Polo, Laia Tenas, Paula Marne, Xavi Gotsens, Tania Menchón, Anna Soteras, Laura Hernandez, Ruth Dominguez, Sandra Pradas, Maria Pascual, Paula Marne, Maria León, Gema Huesa, Marc Vilanova, Sabrina Segundo, Jordi Huguet, and Aleix Sala-Vila.

\section{Funding}

The research leading to these results has received funding from "la Caixa" Foundation. JDG holds a "Ramón y Cajal" fellowship (RYC-2013-13054). CM is supported by the Spanish Ministry of Economy and Competitiveness (grant $n^{\circ}$ IEDI-2016-00690). MS-C received funding from the European Union's Horizon 2020 Research and Innovation Program under Marie Sklodowska-Curie action grant agreement No 752310 .

\section{Availability of data and materials}

The datasets used and/or analyzed during the current study are available from the corresponding author on reasonable request.

\section{Authors' contributions}

All authors listed have made substantial contributions to the conception and design, or acquisition of data, or analysis and interpretation of data. All authors have been involved in the drafting and critical revision of the manuscript and have approved its final version. Authors have agreed to be accountable for all aspects of the work presented here.

Ethics approval and consent to participate

The study was approved by the local ethics committee and all individuals gave written informed consent to participate.

Consent for publication

Not applicable.

\section{Competing interests}

The authors declare that they have no competing interests.

\section{Publisher's Note}

Springer Nature remains neutral with regard to jurisdictional claims in published maps and institutional affiliations.

\section{Author details}

${ }^{1}$ Barcelonaßeta Brain Research Center, Pasqual Maragall Foundation, C/ Wellington 30, 08005 Barcelona, Spain. ${ }^{2}$ CIBER Fragilidad y Envejecimiento Saludable (CIBERFES), Madrid, Spain. ${ }^{3}$ Centro de Investigación Biomédica en Red de Bioingeniería, Biomateriales y Nanomedicina (CIBER-BBN), Madrid, Spain. ${ }^{4}$ Universitat Pompeu Fabra, Barcelona, Spain.

Received: 6 September 2018 Accepted: 18 November 2018 Published online: 20 December 2018

\section{References}

1. Jessen F, Amariglio RE, van Boxtel M, Breteler M, Ceccaldi M, Chételat $G$, et al. A conceptual framework for research on subjective cognitive decline in preclinical Alzheimer's disease. Alzheimers Dement. 2014;10:844-52. 
2. Molinuevo JL, Rabin LA, Amariglio R, Buckley R, Dubois B, Ellis KA, et al. Implementation of subjective cognitive decline criteria in research studies. Alzheimers Dement. 2017;13:296-311.

3. Alegret M, Rodríguez O, Espinosa A, Ortega G, Sanabria A, Valero S, et al. Concordance between subjective and objective memory impairment in volunteer subjects. J Alzheimers Dis. 2015;48:1109-17.

4. Cacciamani F, Tandetnik C, Gagliardi G, Bertin H, Habert M-O, Hampel H, et al. Low cognitive awareness, but not complaint, is a good marker of preclinical Alzheimer's disease. J Alzheimers Dis. 2017;59:753-62.

5. Mendes T, Ginó S, Ribeiro F, Guerreiro M, de Sousa G, Ritchie K, et al. Memory complaints in healthy young and elderly adults: reliability of memory reporting. Aging Ment Health. 2008;12:177-82.

6. Minett TSC, Da Silva RV, Ortiz KZ, Bertolucci PHF. Subjective memory complaints in an elderly sample: a cross-sectional study. Int J Geriatr Psychiatry. 2008;23:49-54.

7. Reid LM, MacLullich AMJ. Subjective memory complaints and cognitive impairment in older people. Dement Geriatr Cogn Disord. 2006;22:471-85.

8. Jessen F, Wiese B, Cvetanovska G, Fuchs A, Kaduszkiewicz H, Kolsch H, et al. Patterns of subjective memory impairment in the elderly: association with memory performance. Psychol Med. 2007;37:1753-62.

9. Koppara A, Frommann I, Polcher A, Parra MA, Maier W, Jessen F, et al. Feature binding deficits in subjective cognitive decline and in mild cognitive impairment. Tales A, Jessen F, Butler C, Wilcock G, Phillips J, Bayer T, editors. J Alzheimers Dis. 2015;48 Suppl 1:S161-S170.

10. Hu X, Uhle F, Fliessbach K, Wagner M, Han Y, Weber B, et al. Reduced future-oriented decision making in individuals with subjective cognitive decline: a functional MRI study. Alzheimers Dement. 2017;6:222-31.

11. Hsu Y-H, Huang C-F, Tu M-C, Hua M-S. Prospective memory in subjective cognitive decline: a preliminary study on the role of early cognitive marker in dementia. Alzheimer Dis Assoc Disord. 2015;29:229-35.

12. Rabin LA, Chi SY, Wang C, Fogel J, Kann SJ, Aronov A. Prospective memory on a novel clinical task in older adults with mild cognitive impairment and subjective cognitive decline. Neuropsychol Rehabil. 2014;24:868-93.

13. Rabin LA, Wang C, Katz MJ, Derby CA, Buschke H, Lipton RB. Predicting Alzheimer's disease: neuropsychological tests, self-reports, and informant reports of cognitive difficulties. J Am Geriatr Soc. 2012;60:1128-34.

14. Reisberg B, Shulman MB, Torossian C, Leng L, Zhu W. Outcome over seven years of healthy adults with and without subjective cognitive impairment. Alzheimers Dement. 2010;6:11-24.

15. Mitchell AJ, Beaumont H, Ferguson D, Yadegarfar M, Stubbs B. Risk of dementia and mild cognitive impairment in older people with subjective memory complaints: meta-analysis. Acta Psychiatr Scand. 2014;130:439-51.

16. Koppara A, Wagner M, Lange C, Ernst A, Wiese B, König H-H, et al. Cognitive performance before and after the onset of subjective cognitive decline in old age. Alzheimer's Dement Diagn Assess Dis Monit. 2015;1:194-205.

17. Eckerström $M$, Göthlin $M$, Rolstad $S$, Hessen E, Eckerström C, Nordlund A, et al. Longitudinal evaluation of criteria for subjective cognitive decline and preclinical Alzheimer's disease in a memory clinic sample. Alzheimer's Dement Diagn Assess Dis Monit. 2017;8:96-107.

18. Jessen F, Feyen L, Freymann K, Tepest R, Maier W, Heun R, et al. Volume reduction of the entorhinal cortex in subjective memory impairment. Neurobiol Aging. 2006;27:1751-6.

19. van der Flier WM, van Buchem $M$ a, Weverling-Rijnsburger AWE, Mutsaers ER, ELEM B, Admiraal-Behloul F, et al. Memory complaints in patients with normal cognition are associated with smaller hippocampal volumes. J Neurol. 2004;251:671-5.

20. Saykin AJ, Wishart HA, Rabin LA, Santulli RB, Flashman LA, West JD, et al. Older adults with cognitive complaints show brain atrophy similar to that of amnestic MCl. Neurology. 2006;67:834-42.

21. Striepens N, Scheef L, Wind A, Meiberth D, Popp J, Spottke A, et al. Interaction effects of subjective memory impairment and ApoE4 genotype on episodic memory and hippocampal volume. Psychol Med. 2011;41:1997-2006.

22. Scheef L, Spottke A, Daerr M, Joe A, Striepens N, Kölsch H, et al. Glucose metabolism, gray matter structure, and memory decline in subjective memory impairment. Neurology. 2012;79:1332-9.

23. Perrotin A, Desgranges $B$, Landeau B, Mézenge F, La Joie R, Egret $S$, et al. Anosognosia in Alzheimer disease: disconnection between memory and self-related brain networks. Ann Neurol. 2015;78:477-86.

24. Schultz SA, Oh JM, Koscik RL, Dowling NM, Gallagher CL, Carlsson CM, et al. Subjective memory complaints, cortical thinning, and cognitive dysfunction in middle-aged adults at risk for AD. Alzheimers Dement. 2015;1:33-40.
25. Meiberth D, Scheef L, Wolfsgruber S, Boecker H, Block W, Träber F, et al. Cortical thinning in individuals with subjective memory impairment. J Alzheimers Dis. 2015;45:139-46.

26. Hafkemeijer A, Altmann-Schneider I, Oleksik AM, van de Wiel L, Middelkoop HAM, van Buchem MA, et al. Increased functional connectivity and brain atrophy in elderly with subjective memory complaints. Brain Connect. 2013; 3:353-62.

27. Stone J, Pal S, Blackburn D, Reuber M, Thekkumpurath P, Carson A. Functional (psychogenic) cognitive disorders: a perspective from the neurology clinic. J Alzheimers Dis. 2015;48:S5-17.

28. Perrotin A, La Joie R, de La Sayette V, Barré L, Mézenge F, Mutlu J, et al. Subjective cognitive decline in cognitively normal elders from the community or from a memory clinic: differential affective and imaging correlates. Alzheimers Dement. 2016;13:550-60.

29. Abdelnour C, Rodríguez-Gómez O, Alegret M, Valero S, Moreno-Grau S, Sanabria Á, et al. Impact of recruitment methods in subjective cognitive decline. J Alzheimers Dis. 2017:57:625-32.

30. Snitz BE, Wang T, Cloonan YK, Jacobsen E, Chang C-CH, Hughes TF, et al. Risk of progression from subjective cognitive decline to mild cognitive impairment: the role of study setting. Alzheimers Dement. 2018;14:734-42.

31. Molinuevo J, Gramunt N, Gispert J, Fauria K, Esteller M, Minguillon C, et al. The ALFA project: a research platform to identify early pathophysiological features of Alzheimer's disease. Alzheimers Dement. 2016:1-11.

32. Rami L, Mollica MA, García-Sanchez C, Saldaña J, Sanchez B, Sala I, et al. The Subjective Cognitive Decline Questionnaire (SCD-Q): a validation study. J Alzheimers Dis. 2014;41:453-66.

33. Buschke H. Rationale of the Memory Binding Test. In: Nilsson LG, Ohta N, editors. Dementia and memory. Hove, East Sussex: Psychology Press; 2014. p. 55-71.

34. Gramunt N, Sánchez-Benavides G, Buschke H, Diéguez-Vide F, PeñaCasanova J, Masramon X, et al. The Memory Binding Test: development of two alternate forms into Spanish and Catalan. J Alzheimers Dis. 2016; 52:283-93.

35. Wechsler D. Escala de Inteligencia Wechsler para adultos IV (Spanish version). Madrid: Pearson; 2012.

36. Goldberg D, Bridges K, Duncan-Jones P, Grayson D. Detecting anxiety and depression in general medical settings. BMJ. 1988;297:897-9.

37. Genin E, Hannequin D, Wallon D, Sleegers K, Hiltunen M, Combarros O, et al. APOE and Alzheimer disease: a major gene with semi-dominant inheritance. Mol Psychiatry. 2011;16:903-7.

38. Fischl B. FreeSurfer. Neurolmage. 2012;62:774-81.

39. Cacciaglia R, Molinuevo JL, Falcón C, Brugulat-Serrat A, Sánchez-Benavides $\mathrm{G}$, Gramunt N, et al. Effects of APOE- $\varepsilon 4$ allele load on brain morphology in a cohort of middle-aged healthy individuals with enriched genetic risk for Alzheimer's disease. Alzheimer's Dement. 2018:14:902-12.

40. Rabin LA, Smart CM, Crane PK, Amariglio RE, Berman LM, Boada M, et al. Subjective cognitive decline in older adults: an overview of self-report measures used across 19 international research studies. J Alzheimers Dis. 2015;48(Suppl 1):S63-86.

41. Hao L, Wang X, Zhang L, Xing Y, Guo Q, Hu X, et al. Prevalence, risk factors, and complaints screening tool exploration of subjective cognitive decline in a large cohort of the Chinese population. J Alzheimers Dis. 2017;60:371-88.

42. Tobiansky R, Blizard R, Livingston G, Mann A. The Gospel Oak Study stage IV: the clinical relevance of subjective memory impairment in older people. Psychol Med. 1995;25:779-86.

43. Squire LR. Memory systems of the brain: a brief history and current perspective. Neurobiol Learn Mem. 2004;82:171-7.

44. Buckley RF, Hanseeuw B, Schultz AP, Vannini P, Aghjayan SL, Properzi MJ, et al. Region-specific association of subjective cognitive decline with tauopathy independent of global $\beta$-amyloid burden. JAMA Neurol. 2017;74:1455.

45. Fernández-Blázquez MA, Ávila-Villanueva M, Maestú F, Medina M. Specific features of subjective cognitive decline predict faster conversion to mild cognitive impairment. J Alzheimers Dis. 2016;52:271-81.

46. Gramunt N, Sánchez-Benavides G, Buschke H, Lipton RB, Masramon X, Gispert JD, et al. Psychometric properties of the Memory Binding Test: testretest reliability and convergent validity. J Alzheimers Dis. 2016;50:999-1010.

47. Strauss E, Sherman EMS, Spreen O. A compendium of neuropsychological tests: administration, norms, and commentary. New York City, New York: Oxford University Press; 2006.

48. Donohue MC, Sperling RA, Salmon DP, Rentz DM, Raman R, Thomas RG, et al. The preclinical Alzheimer cognitive composite: measuring amyloidrelated decline. JAMA Neurol. 2015;71:961-70. 
49. Carr DB, Gray S, Baty J, Morris JC. The value of informant versus individual's complaints of memory impairment in early dementia. Neurology. 2000;55: 1724-6.

50. Cacciottolo M, Wang X, Driscoll I, Woodward N, Saffari A, Reyes J, et al. Particulate air pollutants, APOE alleles, and their contributions to cognitive impairment in older women and to amyloidogenesis in experimental models. Transl Psychiatry. 2017;7:e1022

51. Valech N, Mollica MA, Olives J, Tort A, Fortea J, Lleo A, et al. Informant's perception of subjective cognitive decline helps to discriminate preclinical Alzheimer's disease from normal aging. J Alzheimers Dis. 2015;48:S87-98.

52. Balash Y, Mordechovich M, Shabtai H, Giladi N, Gurevich T, Korczyn AD. Subjective memory complaints in elders: depression, anxiety, or cognitive decline? Acta Neurol Scand. 2013;127:344-50.

53. Peckham AD, McHugh RK, Otto MW. A meta-analysis of the magnitude of biased attention in depression. Depress Anxiety. 2010;27:1135-42.

54. Rabin LA, Smart CM, Amariglio RE. Subjective cognitive decline in preclinical Alzheimer's disease. Annu Rev Clin Psychol. 2017;13:369-96

55. Gispert JD, Rami L, Sánchez-Benavides G, Falcon C, Tucholka A, Rojas S, et al. Nonlinear cerebral atrophy patterns across the Alzheimer's disease continuum: impact of APOE4 genotype. Neurobiol Aging. 2015;36:2687-701.

Ready to submit your research? Choose BMC and benefit from:

- fast, convenient online submission

- thorough peer review by experienced researchers in your field

- rapid publication on acceptance

- support for research data, including large and complex data types

- gold Open Access which fosters wider collaboration and increased citations

- maximum visibility for your research: over $100 \mathrm{M}$ website views per year

At $\mathrm{BMC}$, research is always in progress.

Learn more biomedcentral.com/submissions 